\title{
Endoscopic submucosal dissection of poorly differentiated carcinoma mimicking adenoid-cystic carcinoma of the esophagus
}

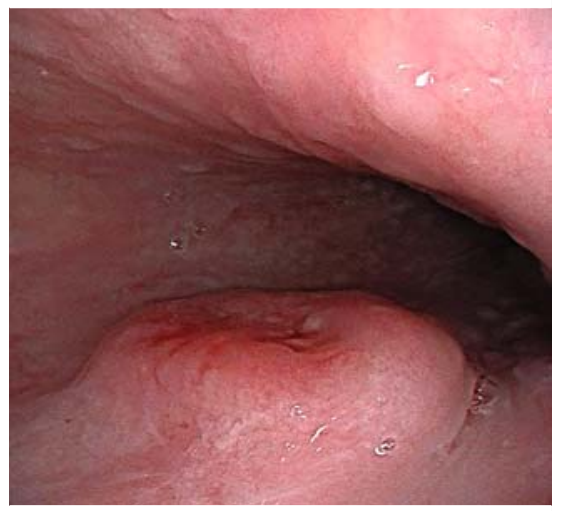

- Fig. 1 Esophagogastroduodenoscopy showed a flat elevated lesion of $15 \mathrm{~mm}$ with a mild central depression in the middle esophagus.

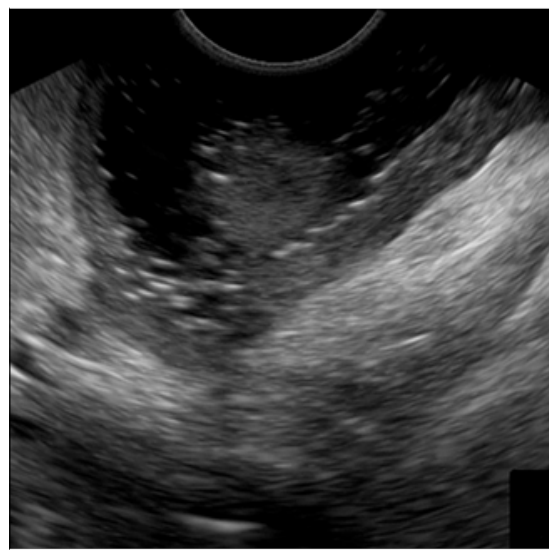

Fig. 2 Endoscopic ultrasound showed the lesion was limited to the mucosal layer.

A 71-year-old man with a history of cryptogenic cirrhosis and hepatocellular carcinoma treated with radiofrequency ablation therapy underwent esophagogastroduodenoscopy (EGD) to evaluate portal hypertension. EGD showed a flat elevated lesion of $15 \mathrm{~mm}$ with a mild central depression ( $>$ Fig.1) and hard consistency on biopsy sampling in the middle esophagus. No esophageal varices were found. The initial histological examination was compatible with ade-
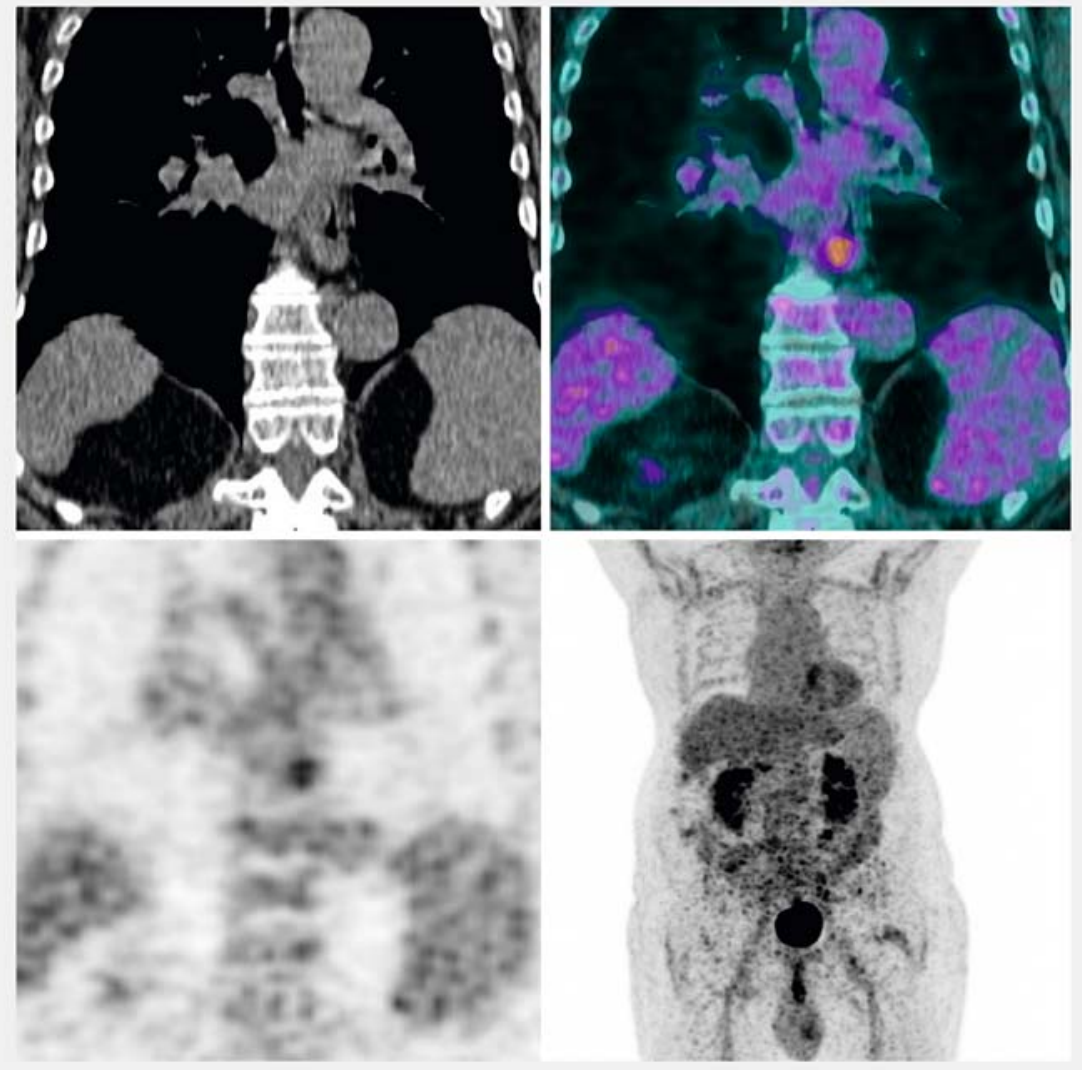

Fig. 3 Staging of the tumor performed with an 18F-fluorodeoxyglucose positron emission tomography/computed tomography: uptake only in the middle tract of the esophagus (standardized uptake value 3.3)

noid cystic carcinoma with a solid pattern.

Adenoid cystic carcinoma is a malignant epithelial tumor arising in the submucosal glands, commonly in the salivary glands and upper respiratory tract. It occurs extremely rarely in the esophagus, where its behavior is biologically aggressive [1]. However, endoscopic ultrasound (EUS) showed a lesion limited to the mucosal layer (> Fig.2). Staging was performed with an 18F-fluorodeoxyglucose positron emission tomography/computed tomography (18F-FDG PET/CT) scan ( Fig.3), which showed only uptake in the middle tract of the esophagus.

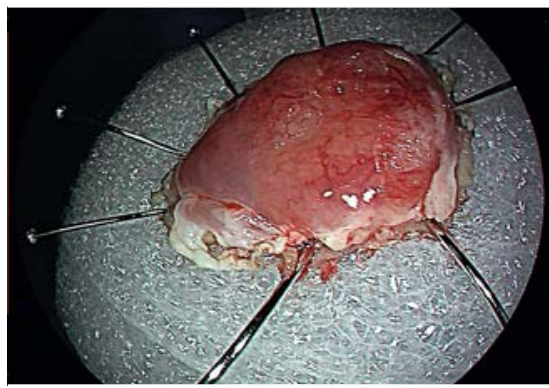

- Fig. 4 En bloc resection was performed by endoscopic submucosal dissection.

Owing to the comorbidities, the patient was judged unsuitable for surgery. Therefore, an en bloc resection ( $>$ Fig. 4) 


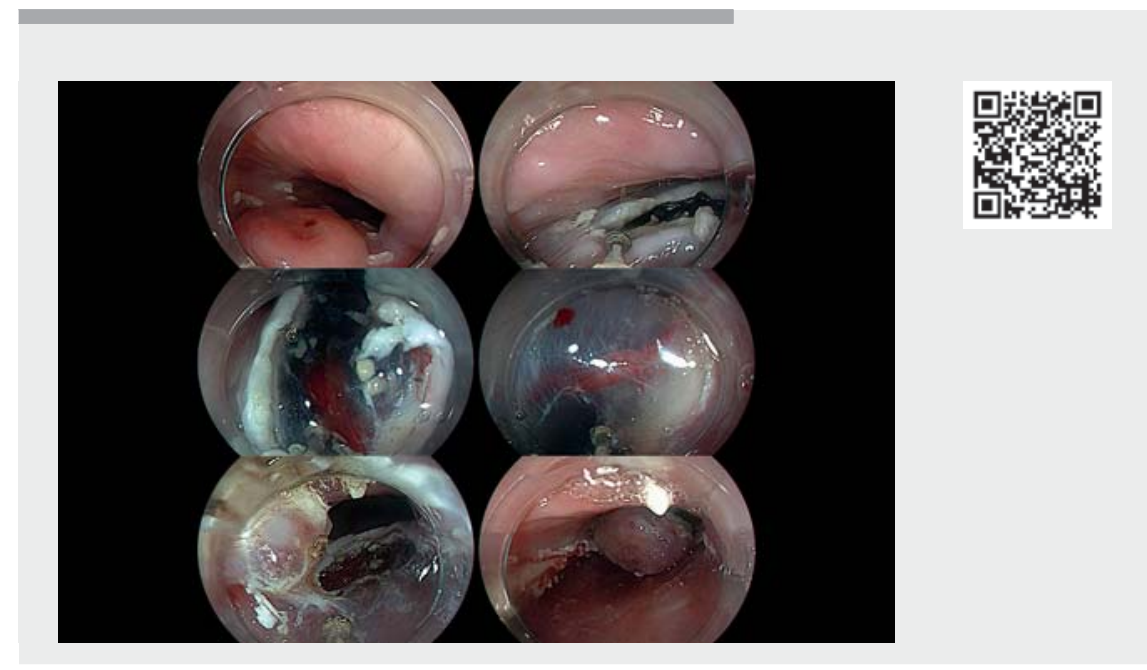

$\checkmark$ Video 1 Esophageal endoscopic submucosal dissection. The lesion, initially typified as adenoid-cystic carcinoma, was in fact a poorly differentiated carcinoma.
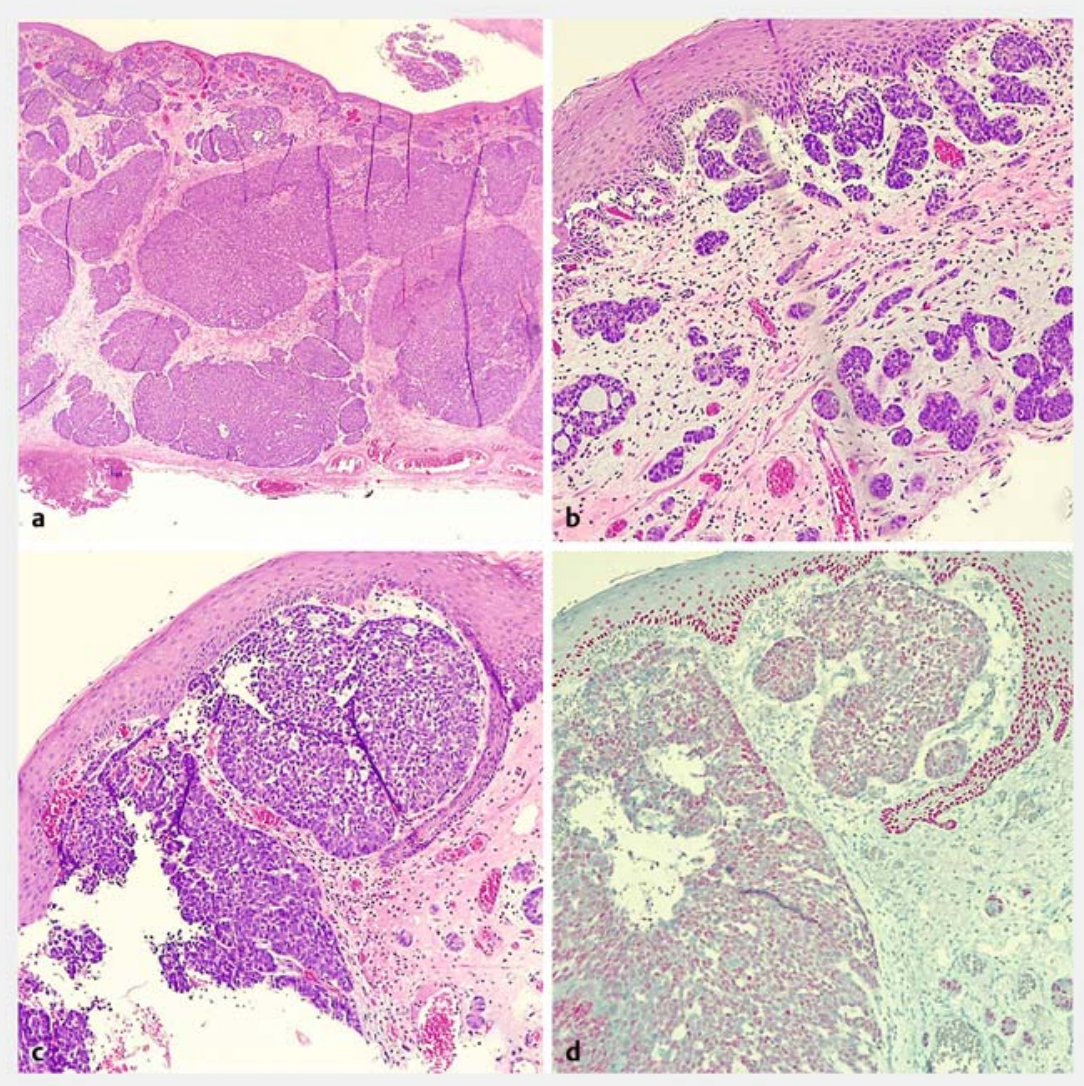

- Fig. 5 Esophageal mucosa with poorly differentiated carcinoma with high proliferative activity (mitotic index: $36 \times 10$ high-power fields), solid pattern with multiple nodular areas separated by fibrous stroma, with glandular aspects, focal necrosis and areas of stromal hyalinization. a $2 \times$ magnification. b $10 \times$ magnification. c $20 \times$ magnification. d Immunohistochemistry: $\mathrm{p} 40+$.

was performed by endoscopic submucosal dissection (ESD) ( $\triangleright$ Video 1). The definitive histological evaluation showed a multidisciplinary evaluation of the case considered only close radiological and endoscopic follow-up indicated.

Endoscopic control at 6 months showed the presence of a regular scar at the site of the previous ESD, with no signs of residual or disease recurrence. At the same time, EUS and CT scan ruled out signs of disease recurrence or metastasis.

Endoscopy_UCTN_Code_TTT_1AO_2AG

Competing interests

The authors declare that they have no conflict of interest.

The authors

Fabio De Vincentis ${ }^{1}$, Roberta Maselli²,3, Mario Brancaccio $^{1}$, Alessandro Mussetto ${ }^{1}$

1 Gastroenterology Unit, Azienda Unità Sanitaria Locale della Romagna, Santa Maria delle Croci Hospital, Ravenna, Italy

2 Department of Biomedical Sciences, Humanitas University, Pieve Emanuele, Milan, Italy

3 IRCCS Humanitas Research Hospital, Rozzano, Milan, Italy

Corresponding author

\section{Fabio De Vincentis, MD}

Gastroenterology Unit, Santa Maria delle Croci Hospital, Viale Vincenzo Randi, 5, 48121 Ravenna, Italy

fabiodevincentis@gmail.com

\section{Reference}

[1] Suzuki H, Nagayo T. Primary tumors of the esophagus other than squamous cell carcinoma-histologic classification and statistics in the surgical and autopsied materials in Japan. Int Adv Surg Oncol 1980; 3: 73-109

\section{Bibliography}

Endoscopy 2022; 54: E619-E621

DOI 10.1055/a-1724-7162

ISSN 0013-726X

published online 26.1.2022

(C) 2022. Thieme. All rights reserved.

Georg Thieme Verlag KG, Rüdigerstraße 14,

70469 Stuttgart, Germany 
CORRECTION

Endoscopic submucosal dissection

of poorly differentiated carcinoma

mimicking adenoid-cystic carcinoma

of the esophagus

De Vincentis F, Maselli R, Brancaccio M.

Endoscopic submucosal dissection of

poorly differentiated carcinoma

mimicking adenoid-cystic carcinoma

of the esophagus.

Endoscopy 2022, doi:10.1055/a-1724-

7162

In the above-mentioned article, the institutions of Roberta Maselli have

been corrected. This was corrected in

the online version on April 14, 2022. 